\title{
Cartílago articular: Evaluación por resonancia magnética
}

\section{Dr. Gonzalo Delgado P.}

Médico Radiólogo. Departamento Imágenes, Clínica Alemana de Santiago. Chile.

Facultad de Medicina Clínica Alemana - Universidad del Desarrollo. Chile.

\begin{abstract}
This article reviews the radiographic evaluation of articular cartilage lesions with emphasis on its magnetic resonance imaging study, we will discuss the usefulness of conventional sequences and advanced MRI studies which allow detection of incipient intrasubstance chondral lesions, prior to the ulceration of its surface.
\end{abstract}

Keywords: Articular cartilage, Magnetic resonance.

Resumen: El presente artículo revisa la evaluación imagenologica de las lesiones del cartílago articular con énfasis en su estudio por resonancia magnética, discutiendo la utilidad de las secuencias convencionales y los estudios avanzados de RM que permiten detectar lesiones condrales incipientes intrasustancia, previo a la ulceración de su superficie.

Palabras clave: Cartílago articular, Resonancia magnética.

Delgado G. Cartílago articular: Evaluación por resonancia magnética. Rev Chil 2013; 19(3): 134-139.

Correspondencia: Dr. Gonzalo Delgado P. / delgado.gonzalo@gmail.com

Artículo y publicado en http://www.alemana.cl/contactocientifico/1/ediciones_anteriores.html

y autorizado para publicación en Revista Chilena de Radiología por ambos editores.

Las lesiones de los cartílagos articulares, son frecuentes en las distintas articulaciones y sus etiologías multifactoriales, incluyendo causas traumáticas, artropatías inflamatorias, infecciosas (artritis séptica) y causas degenerativas. Las lesiones de origen degenerativo son las más frecuentes, siendo un problema de salud pública importante por el alto costo económico y social que representan los gastos directos o indirectos en relación al tratamiento y ausencia laboral. Se calcula que aproximadamente el $75 \%$ de la población mayor de 75 años presenta $\operatorname{artrosis}^{(1)}$.

El cartílago articular o cartílago hialino, es de vital importancia en las articulaciones de tipo diartrosis (articulaciones con amplio rango de movimiento) y sus funciones principales son disipar y transmitir las fuerzas sobre las superficies articulares, amortiguar las cargas y proveer una superficie de deslizamiento adecuada entre las superficies articulares. Como características principales, el cartílago hialino es un tejido avascular (se nutre a través del líquido sinovial), no tiene inervación y no tiene capacidad de regenerarse con el mismo tejido, solo presenta capacidad reparativa limitada con fibrocartílago, el que es de menor resistencia.

El cartílago articular se compone de (Figura 1):

A. Agua (65-80\%): está presente en mayor cantidad en las porciones superficiales del cartílago y su contenido aumenta con el proceso de envejecimiento y en las alteraciones degenerativas.

B. Colágeno (10-20\%): el colágeno que predomina es el tipo II (95\%), corresponde a la matriz de sostén del cartílago y provee resistencia a las fuerzas de tensión. El colágeno es el principal componente en el cartílago deshidratado.

C. Proteoglicanos (10-15\%): son producidos por los condrocitos, siendo sus subunidades, los glicosaminoglicanos (GAG). Proveen resistencia a las fuerzas de compresión y tienen resistencia elástica.

D. Condrocitos (5\%): corresponden a la parte celular del cartílago y son los encargados de producir los proteoglicanos, el colágeno, las proteínas y algunas enzimas. 


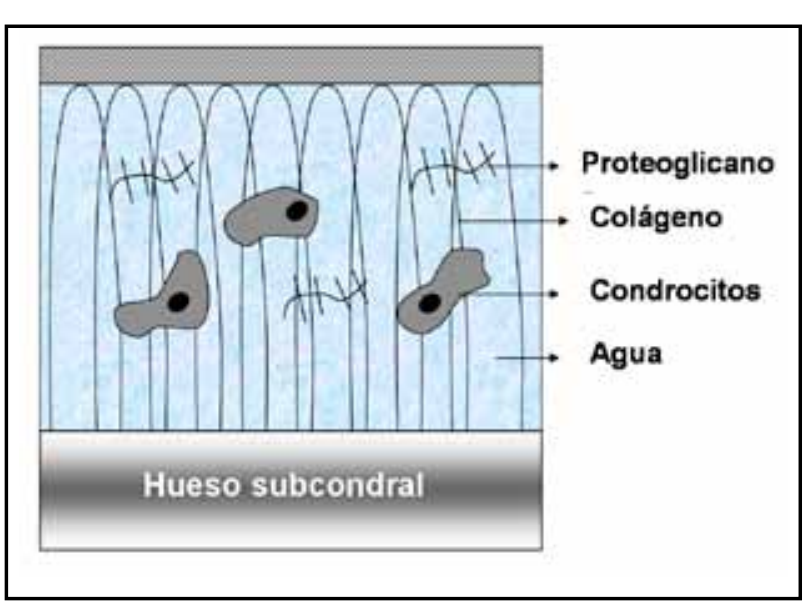

Figura 1. Esquema que muestra los distintos componentes del cartílago articular.

En el cartílago articular se reconocen distintas porciones dependiendo de la profundidad y orientación que adquieren las fibras de colágeno (Figura 2). Así, se reconoce una porción superficial que abarca aproximadamente el 10 a $20 \%$ del grosor del cartílago, donde las fibras de colágeno se disponen en forma paralela a la superficie del cartílago. La porción transicional que corresponde al $40-60 \%$, donde las fibras de colágeno tienen una disposición aleatoria. En la porción radial correspondiente al $30 \%$ aproximadamente, las fibras de colágeno tienen una disposición perpendicular a la superficie y es la porción donde el entrelazado de colágeno es más compacto. Por último, la lámina calcificada corresponde a la zona donde el cartílago se fusiona a la cortical articular ósea.

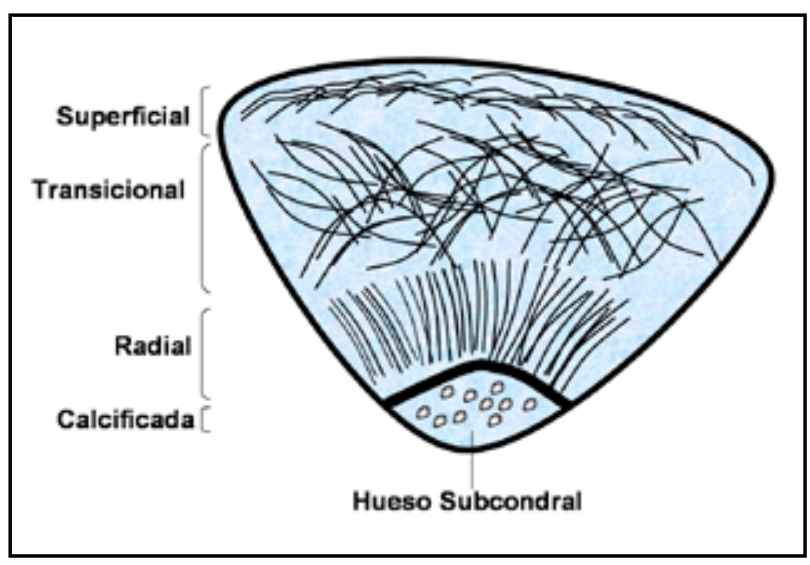

Figura 2. Esquema de las distintas porciones del cartílago articular, dependiendo de la orientación de las fibras de colágeno.

Los procesos de envejecimiento y degeneración del cartílago articular, se asocian a pérdida de la capacidad reproductiva de los condrocitos y disminución de los proteoglicanos. El cartílago se hace más rígido y aumenta su contenido de agua. Estas alteraciones en conjunto determinan una pérdida de las características y funciones del cartílago, haciéndolo menos resistente y proclive a lesionarse con ulceraciones y fisuras.

Se pueden encontrar varias clasificaciones de lesiones condrales, tanto del punto de vista imagenológico como artroscópico. En este aspecto es importante la comunicación con los clínicos, especialmente traumatólogos, debiendo los radiólogos usar clasificaciones conocidas y usadas por los clínicos que interactúan con nosotros y si es necesario adecuar las clasificaciones fundamentalmente artroscópicas a nuestros informes imagenológicos. Una de las clasificaciones más utilizada por los clínicos dedicados al tema de las lesiones condrales, es la clasificación de la ICRS (International Cartilage Repair Society) (Tabla I). Esta clasificación artroscópica es fácil de extrapolar a nuestros estudios de imagen, pues se basa fundamentalmente en la profundidad de la lesión.

Tabla I. Clasificación ICRS (International Cartilage Repair Society) de las lesiones condrales.

$\begin{aligned} 0 & \text { Normal } \\ \text { 1A } & \text { Fibrilación superficial o resblandecimiento } \\ 1 \mathrm{~B} & \text { Fisuras superficiales y laceraciones } \\ 2 & \text { Defecto }<50 \% \\ 3 \mathrm{~A} & \begin{array}{l}\text { Defecto }>50 \% \text { sin alcanzar lámina } \\ \text { calcificada }\end{array} \\ 3 \mathrm{~B} & \text { Defecto }>50 \% \text { alcanza lámina calcificada } \\ 4 \mathrm{~A} & \begin{array}{l}\text { Defecto total con compromiso de placa } \\ \text { subcondral }\end{array} \\ 4 \mathrm{~B} & \begin{array}{l}\text { Defecto total compromiso profundo a } \\ \text { placa subcondral }\end{array} \\ & \end{aligned}$

La resonancia magnética (RM), es el método de elección para evaluar lesiones del cartílago articular por su carácter no invasivo, alto contraste y capacidad multiplanar.

El rendimiento de la RM en la detección de lesiones condrales, dependerá del equipo que se use, siendo necesario para la evaluación de lesiones de cartílagos articulares contar con resonadores de alto campo, de 1,5 ó 3 Tesla. Es importante usar protocolos y secuencias adecuadas para poder identificar lesiones sutiles (Figura 3). La sensibilidad de la RM es directamente proporcional a la magnitud en cuanto a superficie condral comprometida y profundidad de la lesión ${ }^{(2)}$. Por otro lado, los cartílagos de mayor grosor como los de la rodilla son más fáciles de evaluar que lo cartílagos de aquellas articulaciones más pequeñas. Es importante, siempre y en todas los exámenes de RM articular, buscar en forma dirigida las lesiones condrales, pues es habitual que al revisar retrospectivamente $y$ 
comparar con hallazgos quirúrgicos, se vean lesiones que pasaron desapercibidas en la primera lectura. Las características de las lesiones condrales, especialmente cuando son focales y únicas, que debemos precisar en el informe de la RM se resumen en la Tabla II.
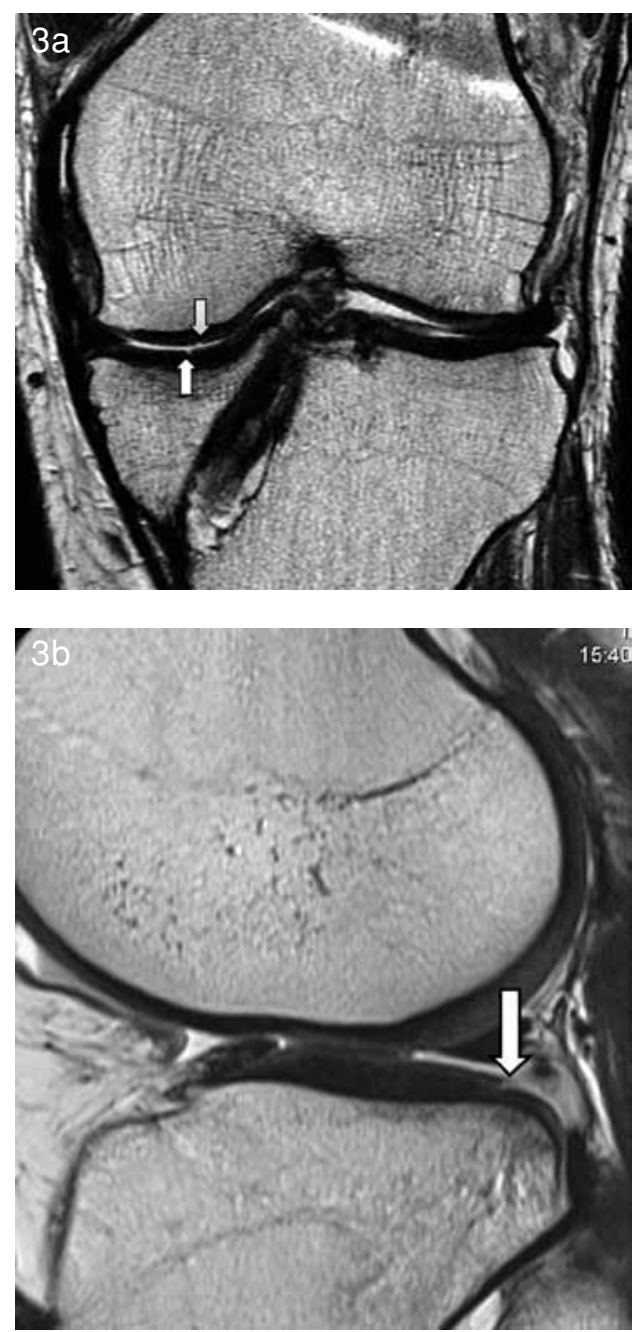

Figura 3. Corte coronal T2 de alta resolución con equipo 1,5 Tesla, muestra pequeñas lesiones superficiales (flechas) en cartílagos del compartimiento medial de la rodilla. Corte sagital T2 en equipo 3 Tesla muestra fina lesión traumática del cartílago del platillo tibial (flecha).

Tabla II. Características importantes de informar en una lesión condral focal.

1. Extensión en superficie midiendo extensión AP y transversal.

2. Profundidad de la lesión (porcentaje del espesor del cartílago comprometido).

3. Ubicación en la superficie articular (compromiso de zona de carga).

4. Alteraciones del hueso subcondral (edema, quistes)

5. Cuerpos condrales u osteocondrales intraarticulares.
Mucho se ha publicado respecto a las secuencias más útiles en la evaluación de cartílagos articulares. Las secuencias con buen contraste entre el cartílago y líquido y con buen contraste entre cartílago y hueso subcondral, son las adecuadas para la evaluación de patología condral. Las secuencias que mejor cumplen con estas condiciones de contraste, son especialmente fast spin echo potenciada en densidad protón (FSE DP) con supresión de grasa y secuencias gradientes potenciadas en T1 (SPGR T1) con saturación de grasa. En FSE DP con saturación de grasa, el cartílago se ve de señal intermedia, el líquido de alta señal y el hueso subcondral de baja señal (Figura 4). En SPGR T1 con supresión de grasa el cartílago se ve de alta señal, y el líquido de baja señal al igual que el hueso subcondral (Figura 5). Esta última secuencia efectuada con técnica 3D, permite realizar cortes finos.

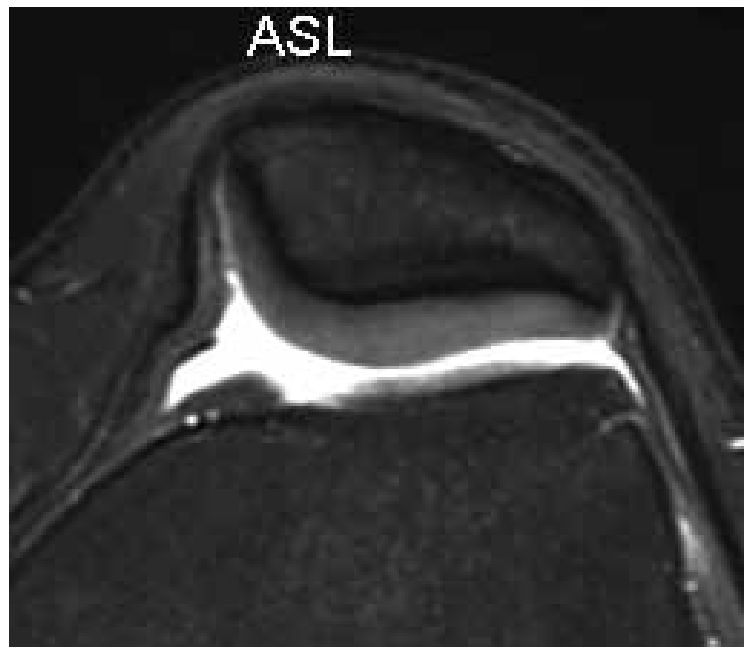

Figura 4. Corte axial potenciado en densidad protón con supresión de la grasa, mostrando el cartílago rotuliano. El cartílago es de señal intermedia contrastando con el líquido articular de alta señal.

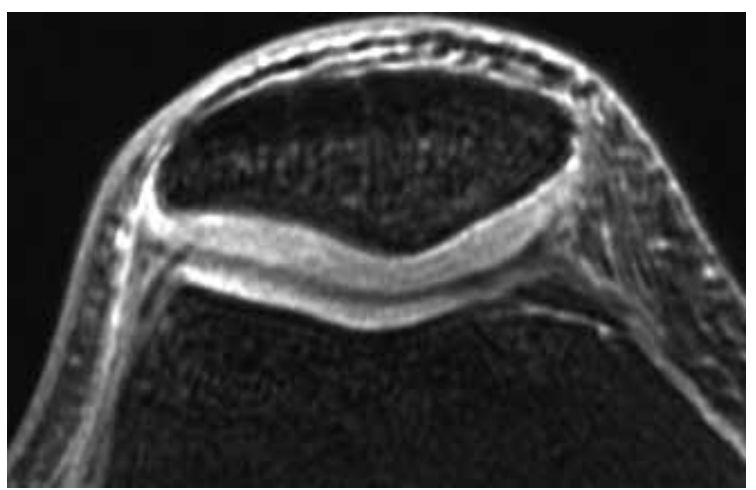

Figura 5. Corte axial en secuencia gradiente SPGR potenciado en $T 1$ mostrando el cartílago rotuliano y de tróclea femoral. El cartílago es de alta señal contrastando con el líquido articular de baja señal. 
Las secuencias T2 presentan buen contraste entre cartílago (baja señal) y líquido (alta señal), sin embargo, el contraste entre cartílago y hueso cortical subcondral no es adecuado, puesto que ambos presentan baja señal. Aunque esta secuencia no es muy sensible a alteraciones sutiles, es importante en las lesiones condrales traumáticas.

Existe bastante consenso en cuanto a que las secuencias más útiles, serían las secuencias FSE DP con supresión de la grasa y SPGR T1 con saturación de grasa ${ }^{(3)}$. Estas secuencias tienen buen contraste y son sensibles a las alteraciones patológicas del cartílago articular lo que las hace las mejores secuencias. Como se mencionó previamente, es importante buscar dirigidamente las lesiones de cartílago independiente del protocolo usado para el estudio.

Las lesiones condrales de origen traumático, son típicamente solitarias y de contornos bien definidos. Con frecuencia comprometen todo el espesor del cartílago (Figura 6) y se pueden asociar a cuerpos osteocondrales o condrales intraarticulares, los que pueden provocar bloqueo articular. Las lesiones de origen degenerativo, se inician con alteraciones bioquímicas intrasustancia, siguiendo con fibrilación, fisuras, ulceraciones de espesor total y por último pérdida de cartílago de espesor total. Habitualmente las lesiones degenerativas son de contornos irregulares y frecuentemente comprometen más de un sector de las superficies articulares con lesiones de espesor variable. Dependiendo de la etapa del compromiso, se pueden observar alteraciones secundarias de artrosis como osteofitos marginales, quistes y alteraciones de señal del hueso subcondral (Figura 7).

La RM es importante en la evaluación de lesiones condrales tratadas en forma quirúrgica. En este sentido las dos técnicas quirúrgicas más frecuentemente usadas son la microfractura y los injertos osteocondrales autólogos. La microfractura consiste en realizar perforaciones en la zona de lesión condral, lo que produce una zona cruenta de hueso subcondral con formación de grupo de células mesenquimales indiferenciadas de la médula ósea que formarán fibrocartílago reparativo, el que es de menor resistencia que el cartílago hialino. Los injertos osteocondrales autólogos fundamentalmente usados en lesiones condrales en superficies de carga de la rodilla, consisten en sacar un fragmento osteocondral en zona de no carga (habitualmente tróclea femoral) para colocarlos en la zona de lesión condral original. Este procedimiento, aunque más demandante técnicamente, tiene la ventaja de reparar la lesión con cartílago hialino.

\section{Estudios avanzados de RM}

Se han desarrollado métodos especiales de RM para evaluación de cartílagos articulares. La mayoría de ellos son estudios que permanecen más bien
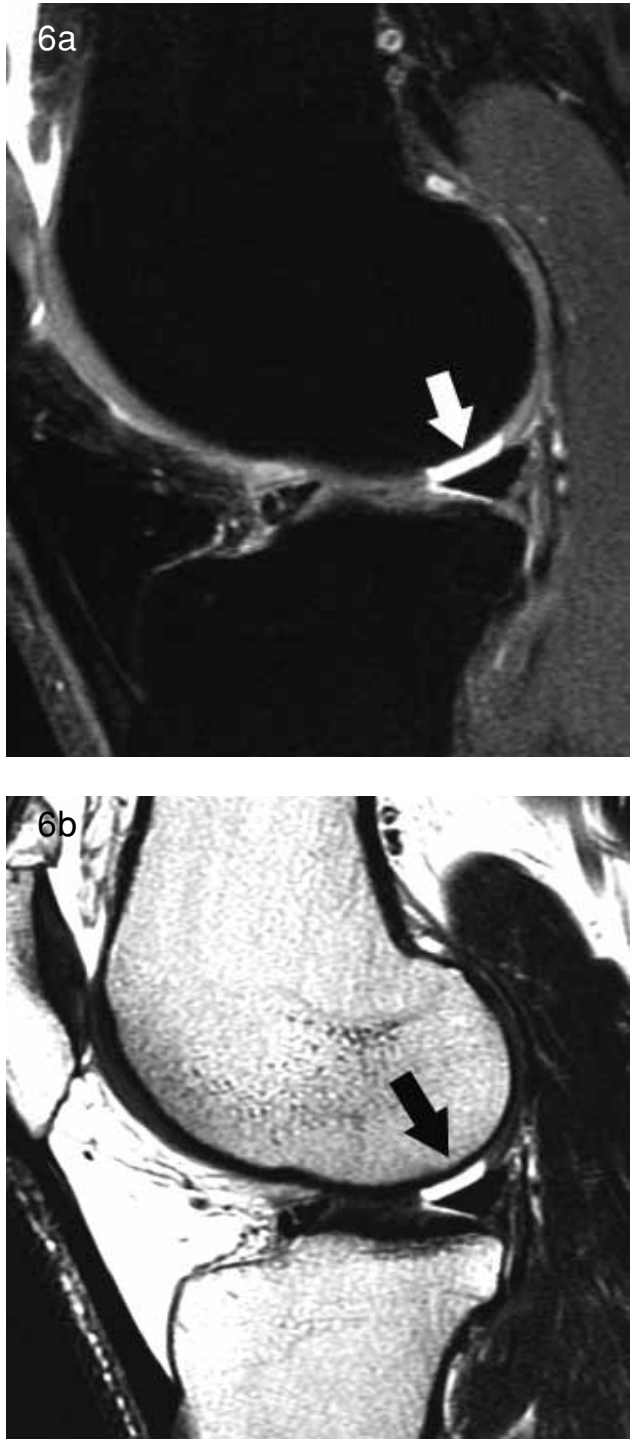

Figura 6. Cortes sagitales del compartimiento femorotibial externo de la rodilla, potenciado en DP con saturación de grasa (a) y T2 (b). Se puede ver una lesión condral focal de espesor total, de origen traumático.

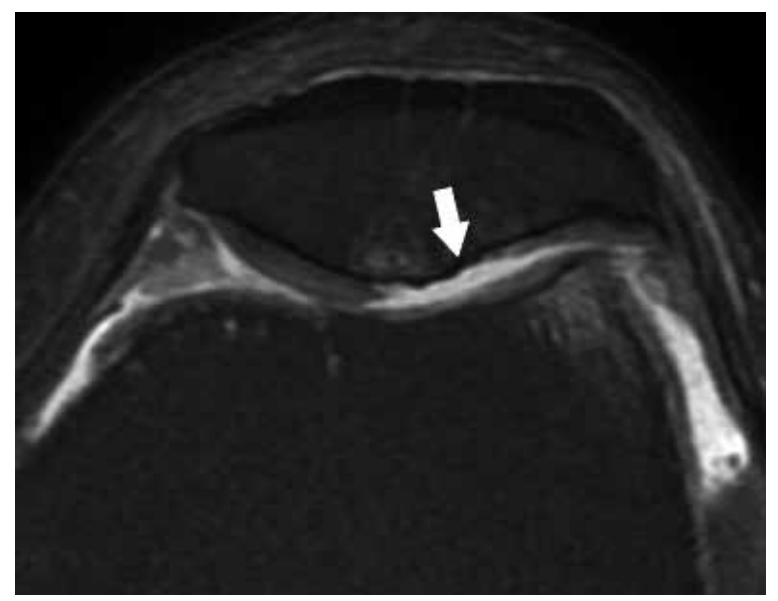

Figura 7. Corte axial potenciado en DP con saturación de grasa, mostrando condropatía degenerativa avanzada de la rótula. 
en investigación, sin aplicación clínica importante, siendo la excepción el estudio mapa T2, el que se ha desarrollado más en la práctica diaria y será revisado con más detalle.

El estudio mapa T2 del cartílago articular, es un método cuantitativo de evaluación de la estructura interna del cartílago. Con esta técnica es posible medir el tiempo de relajación T2 del cartílago.

Los tiempos de relajación T2 en el cartílago normal, son menores en las capas más profundas donde el entrelazado de fibras de cartílago es más compacto y hay menos cantidad de agua. Los tiempos de relajación T2, aumentan hacia las porciones más superficiales del cartílago.

Este método se basa fundamentalmente en que las alteraciones degenerativas producen desorganización de la matriz de colágeno, la que se hace más laxa permitiendo mayor contenido de protones de $\mathrm{H}_{2} \mathrm{O}$ los que además están más libres, lo que produce aumento de los valores de relajación T2, por sobre niveles normales.

Con el programa adecuado, en la estación de trabajo es posible medir el tiempo de relajación T2 en milisegundos, situando el área de interés donde se estime necesario, lo que permite cuantificar objetivamente las alteraciones. Además esto se puede representar morfológicamente en imagen en colores, usando escala predefinida para hacerlo visualmente detectable (Figura 8). Como éste no es un estudio morfológico, sino más bien cuantitativo, su mayor utilidad es detectar alteraciones intrasustancia iniciales del cartílago, previo a las ulceraciones o fisuras de su superficie (Figura 9). Esta capacidad hace que este método pueda evaluar la evolución de alteraciones condrales incipientes después de tratamientos farmacológicos o de otra naturaleza. De las técnicas más avanzadas para evaluación de cartílago, esta es la que se está usando más en la práctica clínica ${ }^{(4-6)}$.

\section{Refuerzo retardado con gadolinio}

Este método consiste en inyectar por vía intravenosa gadolinio iónico, que tiene cargas negativas, y efectuar movilidad activa y ejercicio de la articulación en estudio lo que permitiría paso de contraste hacia el líquido sinovial. Este método permite evaluar la concentración de proteoglicanos en el cartílago $\operatorname{articular}^{(7)}$.

Este estudio se basa en las cargas negativas que tienen los glicosaminoglicanos, que son las subunidades de los proteoglicanos. Se conoce que con los procesos degenerativos y de envejecimiento del cartílago articular, disminuye la cantidad de proteoglicanos. Si existe cantidad normal de glicosaminoglicanos (carga negativa), el contraste (carga negativa) será repelido y no penetrará por difusión al cartílago. Cuando la cantidad de glicosaminoglicanos está disminuida, permite que el contraste penetre e impregne el car-

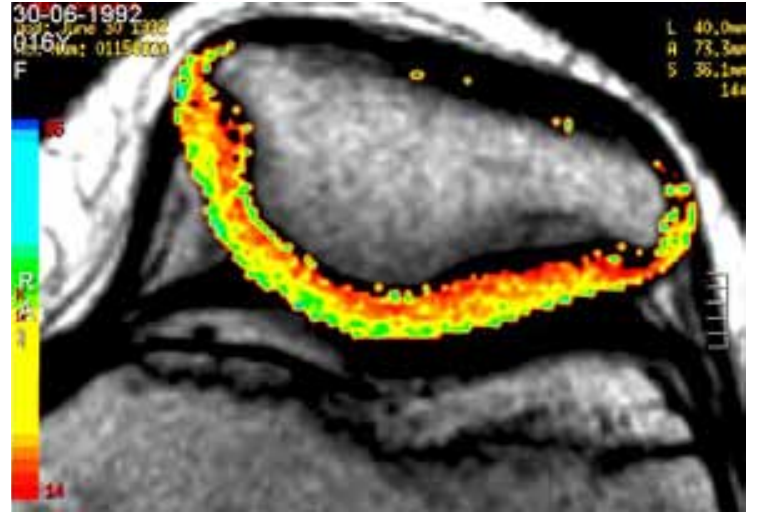

Figura 8. Mapa T2 con escala de colores de cartílago rotuliano normal, donde la parte más profunda muestra coloración roja, indicando niveles bajos de tiempos de relajación T2, la parte central coloración amarilla y la zona más superficial coloración verde indicando niveles más altos de T2.
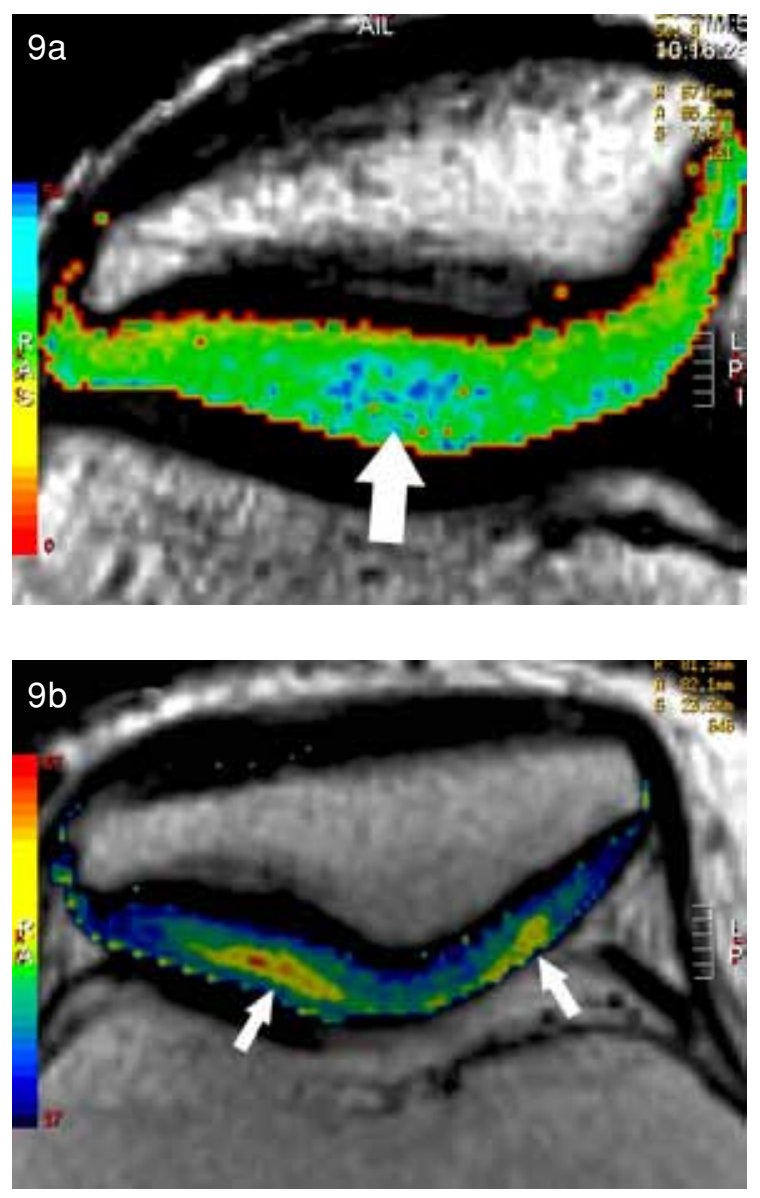

Figura 9. Mapa T2 color en dos pacientes distintos, mostrando zonas alteradas (flechas) con aumento de niveles T2 en el espesor del cartílago articular.

tílago en las zonas alteradas. Esta mayor captación se puede representar en imagen color. 
Dentro de otros métodos avanzados de estudio y fundamentalmente usados en investigación más que en aplicación clínica, podemos mencionar técnicas específicas como proyección de reconstrucción de tiempo corto de eco y espectroscopía de cartílago, entre otros.

\section{Resumen}

El cartílago articular es un tejido altamente resistente. Sin embargo, sus lesiones son frecuentes y la RM es el método de imagen de elección para su evaluación. Para esto son útiles las secuencias convencionales, existiendo también algunas técnicas especializadas de RM que pueden permitir evaluación más objetiva, cuantitativa, de las alteraciones condrales degenerativas incipientes. Varias de estas técnicas especiales están todavía en proceso de desarrollo e investigación y aún no han sido aplicadas a la práctica clínica. Excepción a esto es el estudio mapa T2 de cartílago, el que está siendo usado más rutinariamente.

\section{Bibliografía}

1. Lawrence RC, Hochberg MC, Kelsey JL. Estimate of prevalence of selected arthritic and musculoskeletal diseases in the United States. J Rheumatol 1989; 16(4): 427-441.

2. Figueroa D, Calvo R, Vaisman A, Carrasco M, Moraga $C$, Delgado I. Knee chondral lesions; Incidence and correlation between Arthroscopic and magnetic resonance findings. Arthroscopy 2007; 23(3): 312315.

3. Recht M, Goodwin D, Winalski C, White L.MRI of articular cartilage: Revisiting current status and future directions. AJR 2005; 185: 899-914.

4. Dunn T, Lu Y, Jin H, Ries M, Majumdar S. T2 relaxation times of cartilage at MR Imaging: comparison with severity of knee osteoarthritis. Radiology 2004; 232: 592-598.

5. Mosher T, Dardzinski B, Smith M, et al. Human articular cartilage influence of aging and early symptomatic degeneration on the spatial variation of T2-Preliminary findings at 3T. Radiology 2000; 214: 259-266.

6. Watrin A, Ruaud JP, Olivier P, Guingamp N, et al. T2 mapping of rat patellar cartilage. Radiology 2001; 219: 395-402.

7. Young A, Stanwell P, Williams A, Rohrsheim J, Parker $D$, Giuffre B, et al. Glycosaminoglycan content of knee cartilage following posterior cruciate ligament rupture demonstrated by delayed gadolinium-enhanced magnetic resonance imaging of cartilage (dGEMRIC). Bone and Joint Surg 2005; 87(12): 2763-2767. 